\title{
Most Scottish neurologists do not apply the 2010 McDonald criteria when diagnosing multiple sclerosis
}

\author{
${ }^{1} \mathrm{R}$ Lumley, ${ }^{2} \mathrm{R}$ Davenport, ${ }^{3} \mathrm{~A}$ Williams \\ ${ }^{1}$ Medical Student, University of Edinburgh, Edinburgh; ${ }^{2}$ Consultant Neurologist, Department of Clinical Neurosciences, Western General \\ Hospital, Edinburgh; ${ }^{3}$ Scottish Senior Research Fellow and Honorary Consultant Neurologist, Centre for Regenerative Medicine, University of \\ Edinburgh, Edinburgh, UK
}

\begin{abstract}
Background: The diagnostic criteria for multiple sclerosis have evolved over time and currently the 2010 McDonald criteria are the most widely accepted. These criteria allow the diagnosis of multiple sclerosis to be made at the clinically isolated syndrome stage provided certain criteria are met on a single magnetic resonance brain scan. Our hypothesis was that neurologists in Scotland did not use these criteria routinely.

Method: We sent a SurveyMonkey questionnaire to all Scottish neurologists (consultants and trainees) regarding the diagnosis of multiple sclerosis.

Results: Our questionnaire response rate was 65/99 (66\%). Most Scottish neurologists were aware of the criteria and $31 / 58$ (53\%) felt that they were using these routinely. However, in a clinical vignette designed to test the application of these criteria, only $5 / 57$ (9\%) of neurologists appeared to use them.

Conclusion: Scottish neurologists' use of the 2010 McDonald criteria for diagnosis of multiple sclerosis varies from practitioners' perception of their use of these criteria.
\end{abstract}

KEYWORDS multiple sclerosis, McDonald criteria

DECLARATION OF INTERESTS No conflict of interest declared. AW is funded by a Scottish Senior Research Fellowship

\author{
Correspondence to A Williams \\ Centre for Regenerative Medicine \\ University of Edinburgh \\ 5 Little France Drive \\ Edinburgh EHI 6 UU \\ UK
}

e-mail anna.williams@ed.ac.uk

\section{BACKGROUND}

Multiple sclerosis (MS) is generally of the relapsingremitting type, and thus most patients initially present with an isolated episode of neurological dysfunction, which traditionally has been referred to as a 'clinically isolated syndrome' (CIS). Some of these patients, but not all, will develop MS and this poses a challenge to neurologists and patients, with conversion rates varying from $10-85 \%$ and with highly variable timing of the next episode. Diagnostic criteria for MS have evolved over decades in an attempt to refine this diagnostic process. These criteria have been developed first as research tools, but then adopted as criteria for use in routine clinical practice.Schumacher first proposed MS diagnostic criteria in 1965, ${ }^{2}$ emphasising the key principles of two distinct episodes of neurological dysfunction lasting at least 24 hours, occurring at least one month apart, involving two separate areas of the central nervous system, as confirmed by history or examination. These principles are now better known as dissemination in time (DIT) and space (DIS).
In 1983, Poser incorporated 'paraclinical' testing and cerebrospinal fluid (CSF) oligoclonal bands. ${ }^{3} \mathrm{His}$ criteria allowed 'probable' as well as 'definite' MS diagnoses. In 200 I, McDonald et al. ${ }^{4}$ expanded the role of magnetic resonance (MR) imaging in proving DIS and DIT after a $\mathrm{CIS}$, making it possible to diagnose MS without a second clinical attack, provided new radiological lesions appeared on interval imaging. Revision in $2005^{5}$ included that DIT could be fulfilled on interval imaging within 30 days rather than three months after a CIS. In the latest iteration (2010), ${ }^{6}$ the number of lesions required to fulfill DIS was reduced to at least two out of four anatomical areas and, notably, DIT can be fulfilled at the time of first symptoms after a single MR scan, providing both gadolinium-enhancing and non-enhancing lesions are present. It is suggested that these McDonald criteria are the most sensitive yet and are highly specific for MS. ${ }^{7}$ Most guidelines now include these McDonald criteria as the clinical diagnostic gold standard, including the most recently updated NICE guidelines. ${ }^{8}$ However, clinicians used to the historical clinical criteria requiring two or 
more episodes may be uncomfortable with diagnosing MS after just one clinical episode, and our anecdotal experience was that many neurologists in Scotland were not using the new criteria, preferring the tried and tested clinical 'two or more episodes' rule. Scotland has the highest incidence of $M S$ in the world at 72 in $100,000 /$ year $^{9}$ and thus the condition should be seen frequently by neurologists in Scotland. We therefore audited how neurologists in Scotland diagnose MS in routine clinical practice and to what extent they use the $2010 \mathrm{McD}$ onald criteria.

\section{METHODS}

We designed a questionnaire to audit the use of the McDonald 2010 criteria and other diagnostic tests, including a vignette representing a typical episode of central nervous system symptoms suggestive of a $\mathrm{CIS}$ episode, but specifically designed to allow a diagnosis of MS if applying the 2010 criteria. We used SurveyMonkey to create and distribute the questionnaire (Appendix I, available with the online version of this paper). We targeted all neurologists (consultants and trainees) working in Scotland, using an established Scotland-wide email list for neurologists. The survey was anonymous, voluntary and part of an audit, thus ethical approval was not required. We divided Scotland into four health regions to detect regional differences: South East Scotland (Lothian, Forth Valley, Borders, Fife, Dumfries and Galloway), West of Scotland (Greater Glasgow and Clyde), Grampian and the Highlands (Aberdeen, Inverness), and Tayside (Dundee, Perth). To maintain anonymity we did not ask neurologists whether they had a special interest in MS, as most health regions have only one or two consultants with MS as a sub-specialty interest which would allow their identification.

We used the 2-sample Z-test to compare sample proportions with $p<0.05$ considered statistically significant.

\section{RESULTS}

\section{Demographics}

Of the 99 neurologists working in Scotland, 65 replied (66\%) with 48 (74\%) of these completing the survey fully. None answered less than $80 \%$ of the questions. The majority of respondents were consultants [47/65 (72\%) vs $18 / 65(28 \%)$ trainees]. To give an idea of experience of each respondent we requested the actual or expected Certificate of Completion of Training year, which ranged from 1987-2018. In terms of geographical spread, 28/6 I (46\%) of respondents came from South East Scotland, 15/6| (25\%) from the West of Scotland, 10/61 (16\%) from Grampian and the Highlands and 8/6I (13\%) from Tayside. The highest number of neurologists is in the West, yet only $25 \%$ of respondents came from here, so this group is under-represented. More than $75 \%$ (36/46) of respondents were consultants, except in the West where this number was $53 \%(8 / 15)$.

\section{Familiarity and application of 2010 McDonald criteria}

Almost all [58/60 (97\%)] neurologists were familiar with the 2010 McDonald criteria, ranking their familiarity as 'intimately' [19/60 (32\%)] or 'not in detail' [39/60 (65\%)] (Figure la). Consultants were significantly more likely to be intimately familiar with the criteria compared to trainees [I8/43 (42\%) vs I/I7 (6\%), $p<0.0 \mathrm{I}]$. Around half of respondents $[31 / 58(53 \%)]$ stated that they routinely applied these criteria in clinical practice, and this was influenced by degree of familiarity with the criteria, with those intimately familiar more likely to apply them than those who did not know these criteria in detail [16/19 (84\%) vs $15 / 39$ (38\%), $p<0.01]$. However, there was regional variation: only 10/27 (37\%) routinely applied the criteria in South East Scotland compared to I0/I5 (67\%) in the West, $7 / 10(70 \%)$ in Grampian and the Highlands and $5 / 7(7 \mid \%)$ in Tayside $(p<0.05)$ (Figure $\mid \mathrm{b})$ and this was not accounted for by differences in familiarity with the criteria.

\section{Diagnosing MS}

We found that $36 / 60(60 \%)$ of neurologists would either 'rarely' [23/60 (38\%)] or 'never' [13/60 (22\%)] routinely request a follow-up MRI scan $\geq 30$ days later for a patient presenting with a CIS but with no subsequent symptoms; $4 / 60(7 \%)$ said they would 'usually' request such imaging, 20/60 (33\%) 'sometimes' (Figure Ic). Again, there were regional differences, with repeat imaging more likely to be requested in the West [10/16 (66\%)] and Tayside $[7 / 8(88 \%)]$ than South East Scotland [6/28 (21\%)] or Grampian and the Highlands [1//0 (10\%)]. CSF analysis was 'usually' performed by $22 / 60$ (33\%) of neurologists, with trainees more likely to request lumbar puncture:9/17 (53\%) reported 'usually' requesting lumbar puncture compared to $13 / 43$ (30\%) for consultants $(p<0.05)$. Evoked potentials were seldom used; $34 / 60$ (57\%) of neurologists 'rarely' requested them and $7 / 60$ (I2\%) 'never'.

\section{CIS Vignette}

The results are summarised in Figure Id. The majority [40/55 (73\%)] of neurologists would not request a follow up MR scan, except in Tayside where 6/8 (75\%) of respondents would. Regarding other paraclinical tests, $10 / 15(67 \%)$ of trainees compared to $16 / 41$ (39\%) of consultants would request CSF (similar results to above). More neurologists would request measurement of antinuclear factor [43/56 (77\%)], and antiphospholipid antibodies/lupus anticoagulant [3l/52 (60\%)], but few would request evoked potentials [9/53 (17\%)]. The majority [5।/55 (93\%)] would not recommend disease 


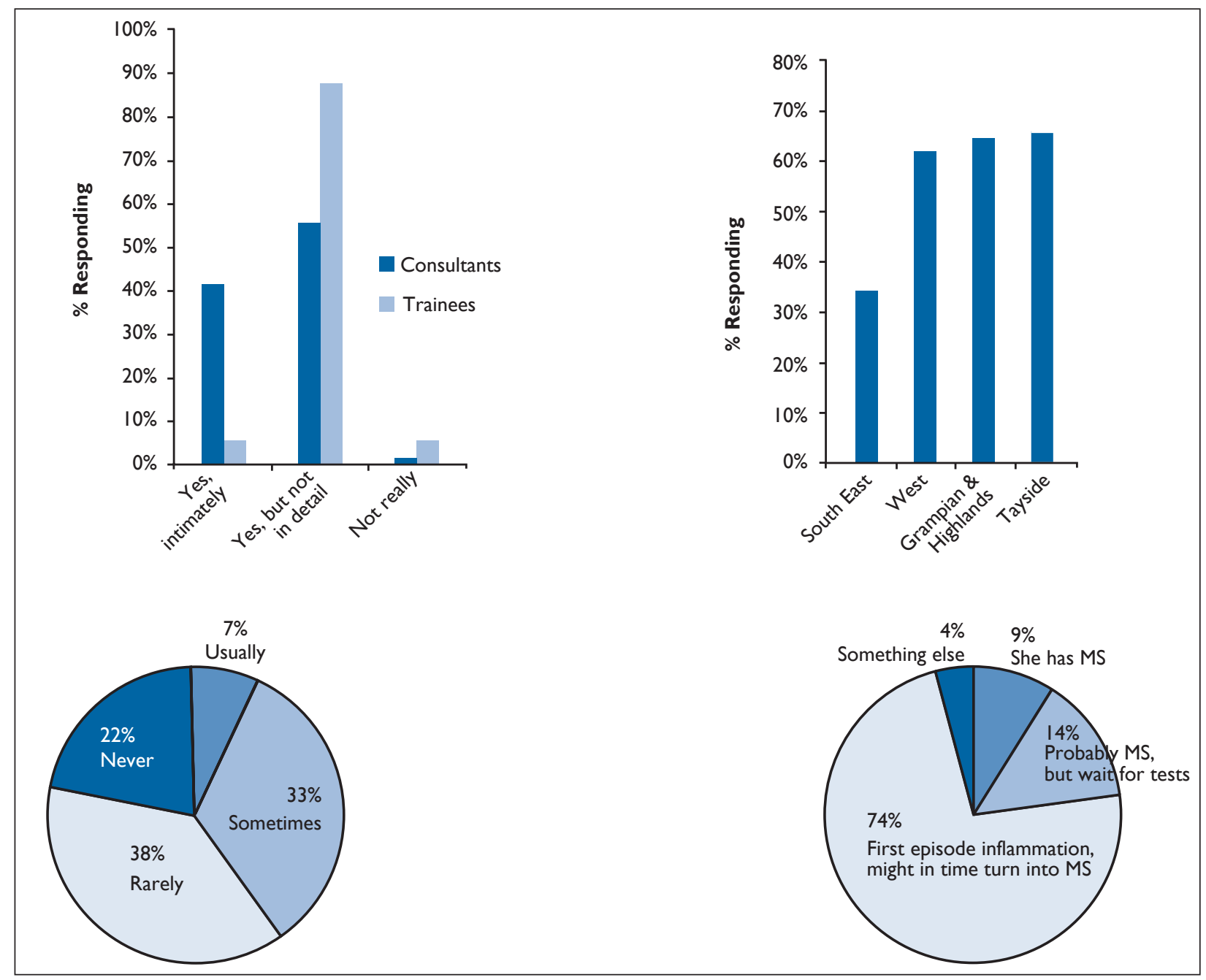

FIGURE I A:Are you familiar with the McDonald 2010 (revised) diagnostic criteria for MS? Most neurologists in Scotland know these criteria, though significantly more consultants consider that they know these in detail compared to trainees ( 2 sample Z-test $* *<<0.01,{ }^{*} p<0.05$ ); B: Do you routinely apply these criteria in your clinical practice? Many neurologists consider that they use these criteria to diagnose MS in practice, but there are regional variations with significantly less neurologists in the South East of Scotland using these criteria compared to other regions (2 sample Z-test, $\left.{ }^{*} p<0.05\right)$; C: Do you order a second MR scan routinely with patient with a first clinical episode suggestive of a CIS with no further symptoms? Despite considering that they use the criteria routinely, most neurologists do not routinely order a second MR brain scan in a patient with a first clinical episode with no further neurological symptoms; $\mathbf{D}$ : Summary of the results of responses to clinical vignette questions; $E$ : What do you tell the vignette patient? In a clinical vignette designed to allow the diagnosis of MS (according to the 2010 McDonald criteria), most respondents did not make the diagnosis of $\mathrm{MS}$, despite more than half $(53 \%)$ considering that they routinely use these criteria

modifying therapy for such a patient. A total of $74 \%$ respondents (42/57) would not make a diagnosis of MS in this case, but would inform the patient that they had a 'first episode of inflammation' that 'could turn into MS' - thus considering this a CIS episode. Only 5/57 (9\%) of all respondents opted for a diagnosis of MS, even though this vignette provides sufficient evidence to do so, according to the 2010 criteria (Figure le).

\section{DISCUSSION}

We found that most Scottish neurologists are not applying the McDonald 2010 criteria and those who believe they do are perhaps not applying it strictly [only
$5 / 57$ (9\%) would diagnose MS in the case vignette although it fulfilled the criteria for a diagnosis of $\mathrm{MS}$, compared to $31 / 58$ (53\%) who said they routinely applied the criteria]. Most Scottish neurologists would not treat what they consider to be a CIS epsiode or make a MS diagnosis from just one scan/clinical episode, and prefer to wait for another relapse rather than order an interval MR scan as evidence for DIT - suggesting a preference for the older, clinically-based criteria than newer MR based ones. Perhaps such caution is justified; one series suggested the sensitivity of a single MR scan after a first clinical episode was $52 \%$, with a specificity of $75 \%,{ }^{10}$ and larger validating studies have not been performed. It may also be the case that, with the advent 
of an increasing range of disease modifying therapies, neurologists are cautious about making a diagnosis after a single episode which might then lead to treatment. (Enthusiasm for disease-modifying therapies after a confirmed CIS or even a first episode of symptoms confirmed as MS in the UK is currently limited due to uncertainty if MS will ensue or if the benefits of treatment outweigh the risk in this situation.)

Some may suggest that Scottish neurologists are conservative and 'behind the times'. For this reason, we examined differences between consultants and trainees, and length of experience, with the hypothesis that trainees or recently qualified consultants may be more 'up-to-date'. However, if anything, trainees were more likely to order other paraclinical tests and less likely to make a diagnosis of MS.We identified regional differences in practice in Scotland, with South East Scotland neurologists being most reluctant to use the new criteria and Tayside neurologists the most enthusiastic; the underlying reason for this is unclear, but probably represents individual preferences rather than any systematic explanation. There is no difference in ability to prescribe disease-modifying therapies between different health boards in Scotland, and so it is unlikely that access to disease-modifying therapies will influence enthusiasm to diagnose MS in this way.

Is it better for patients to receive a diagnosis of MS early? Perhaps, provided it is accurate, given the advent of new MS treatments which significantly reduce relapses. There may also be other benefits; one study indicated that a diagnosis of MS rather than a CIS epsiode reduces anxiety and increases quality of life and 'psychological wellbeing' of patients." Neurologists may be better suited to the uncertainty of a CIS than patients, who may find understanding MS easier for themselves, family and colleagues. However, what evidence there is suggests that if all patients were treated at this point, some would receive long term unnecessary treatment with potential adverse effects which has both medical and financial implications.

We recognise limitations to our study. The sample size was small with only a $66 \%$ response rate. The vignette, while designed to allow a MS diagnosis, was a benign one and perhaps responses would have been different had the clinical episode been more disabling. Nonetheless, the results reflect our anecdotal experience in Scotland (in 2013) and it would be interesting to see if there is similar practice elsewhere. If significant differences in diagnostic approach do exist, in spite of these criteria being nominally adopted, there may be implications for the applicability of MS studies to standard clinical practice. Perhaps Scottish neurologists' diagnostic approach is yet to catch up with that of the latest McDonald criteria, or perhaps the criteria are out of harmony with the reality of Scottish clinical practice.

\section{ACKNOWLEDGEMENTS}

We acknowledge the REMIND fund for funding the SurveyMonkey questionnaire.

\section{REFERENCES}

I Miller DH, Chard DT, Ciccarelli O. Clinically isolated syndromes. Lancet Neurol 2012; II: 157-69. http://dx.doi.org/I0.1016/SI4744422(II)70274-5

2 Schumacker GA, Beebe G, Kibler RF et al. Problems of experimental trials of therapy in multiple sclerosis: report by the panel on the evaluation of experimental trials of therapy in multiple sclerosis. Ann NY Acad Sci 1965; 122: 552-68.

3 Poser CM, Paty DW, Scheinberg $L$ et al. New diagnostic criteria for multiple sclerosis: guidelines for research protocols. Ann Neurol 1983; |3: 227-31.

4 McDonald WI, Compston A, Edan G et al. Recommended diagnostic criteria for multiple sclerosis: guidelines from the International Panel on the diagnosis of multiple sclerosis. Ann Neurol 200I; 50: I2I-7.

5 Polman $\mathrm{CH}$, Reingold SC, Edan G et al. Diagnostic criteria for multiple sclerosis: 2005 revisions to the "McDonald Criteria". Ann Neurol 2005; 58: 840-6.

6 Polman CH, Reingold SC, Banwell B et al. Diagnostic criteria for multiple sclerosis: 2010 revisions to the McDonald criteria. Ann Neurol 201 I; 69: 292-302. http://dx.doi.org/I0.1002/ana.22366

7 Trojano M. Multiple sclerosis: the evolving diagnostic criteria for multiple sclerosis. Nat Rev Neurol 20I I; 7: 25I-2.

8 NICE. Multiple sclerosis: management of multiple sclerosis in primary and secondary care. NICE guidelines [CGI86]; October 2014. http://www.nice.org.uk/guidance/cgl86/chapter/key-priorities-forimplementation (accessed I8/2/20I5).

9 Donnan PT, Parratt JD, Wilson SV et al. Multiple sclerosis in Tayside, Scotland: detection of clusters using a spatial scan statistic. Mult Scler 2005; I I: 403-8.

10 Gómez-Moreno M, Díaz-Sánchez $M$, Ramos-González A. Application of the 2010 McDonald criteria for the diagnosis of multiple sclerosis in a Spanish cohort of patients with clinically isolated syndromes. Mult Scler 2012; 18: 39-44. http://dx.doi. org/I0.II77//3524585 I|4I7828

I I Mattarozzi K,Vignatelli L, Baldin E et al. Effect of the disclosure of MS diagnosis on anxiety, mood and quality of life of patients: a prospective study. Int J Clin Pract 2012; 66: 504-14. http://dx.doi. org/I0.I I I I/j. I742-I24I.20I2.029I2.x 